\title{
Search for single nucleotide polymorphisms (SNPs) associated with hypertension in the genome of senescence-accelerated OXYS rats
}

\author{
Vasiliy A. Devyatkin \\ Laboratory of molecular mechanisms \\ of aging \\ Institute of Cytology and Genetics \\ SB RAS, Novosibirsk, Russia \\ devyatkin@bionet.nsc.ru
}

\author{
Natalia A. Muraleva \\ Laboratory of molecular mechanisms \\ of aging \\ Institute of Cytology and Genetics \\ SB RAS, Novosibirsk, Russia \\ myraleva@bionet.nsc.ru \\ Nataliya Kolosova \\ Laboratory of molecular mechanisms \\ of aging \\ Institute of Cytology and Genetics \\ SB RAS Novosibirsk, Russia \\ kolosova@bionet.nsc.ru
}

\author{
Olga E. Redina \\ Institute of Cytology and Genetics \\ SB RAS, Novosibirsk, Russia \\ oredina@bionet.nsc.ru
}

\begin{abstract}
Aging is a risk factor for many diseases, but the the chance of development with age also depends on genetic factors, environmental conditions, lifestyle and the presence of other pathologies. The OXYS rat strain (ICG SB RAS) is a unique model for studying the mechanisms of aging, since already at an early age these animals develop a whole complex of age-dependent diseases, including cataract, retinopathy, osteoporosis, hypertension and Alzheimer's Disease-like pathology. While hypertension has typical for an age-related disease risk factors, it itself is a risk factor for many other pathologies. However, a complex senile phenotype does not appear in other hypertensive models even with a higher blood pressure. The aim of this study was, based on the results of RNA-Seq, to search for single nucleotide polymorphisms that could contribute to the development of hypertension in senescence-accelerated OXYS rats. We found that OXYS rats are genetically distant from other strains and presumably have their own basis for the development of hypertension, which may determine the absence of senile phenotype of OXYS rats in the hypertensive rat strains.
\end{abstract}

\section{Keywords - hypertension, SNPs, aging, OXYS rats}

\section{Introduction}

Aging is a risk factor for many diseases, but the the chance of development with age also depends on genetic factors, environmental conditions, lifestyle and the presence of other pathologies. Currently, studies on biological models, the genetic uniformity and standard conditions of detention of which increase the reproducibility of the results and reduce the influence of external factors, are a productive approach to elucidating the genetic overlap between age-related diseases. The OXYS rat strain, developed in ICG SB RAS, is a unique model of accelerated aging, which already at early age is characterized by a phenotype similar to geriatric diseases in humans, including cataracts, cardiomyopathy, hypertension, retinopathy and neurodegenerative brain pathology with the signs of Alzheimer's disease [1].

The destructive effect of hypertension on blood vessels, leading to hypoperfusion and metabolic stress, is considered in many studies as a risk factor for cataracts, cardiovascular diseases, osteoporosis and neurodegenerative changes in the brain.
At the age of 3 months in OXYS rats was detected the moderate increase in blood pressure. At the same time the first manifestations of other pathologies in OXYS rats appear. In hypertensive rat strains or induced hypertension models many age-dependent diseases do not occur or manifest in a milder form, in contrast to the complex manifestation in OXYS rats. Its may indicate the importance of the genetic component in the development of age-related diseases.

We previously showed that the genome of the OXYS rat contains a large number of single nucleotide polymorphisms (SNPs) not found in other rat strains. The purpose of this study was to compare the data of transcriptome of OXYS rats with the genotypes of hypertensive rat strains for found the SNPs that could contribute to the development of hypertension in senescence-accelerated OXYS rats.

\section{Methods}

Male OXYS rats were used at ages 20 days and 3, 5, and 18 months, while age-matched male Wistar rats served as controls (3-5 per group). The animals were kept at the Center for Genetic Resources of Laboratory Animals at the ISG SB RAS under standard laboratory conditions $\left(22 \pm 2^{\circ} \mathrm{C}, \quad 60 \%\right.$ relative humidity, $12 \mathrm{~h}$ light $/ 12 \mathrm{~h}$ dark cycle, lights on at 9 a.m.). The food and water were available ad libitum. The protocol of the animal experiment was approved by the Bioethical Committee of the Institute of Cytology and Genetics.

We analyzed RNA-Seq data obtained in early studies for each sample of retinal, prefrontal-cortex, and hippocampal RNA by Illumina nonstranded sequencing (on an Illumina GA IIx at Genoanalytica) in accordance with standard Illumina protocols (mRNA-Seq Sample Prep Kit). The sequencing data were prepared and SNPs positions were identified as described previously [2].

The list of SNPs of OXYS rats was compared with RGSC 6.0 data for genome sequences of 45 rat strains and substrains: 11 of these strains/substrains are commonly employed as a normotensive control (FHL/EurMcwi, LN/MavRrrc, LL/MavRrrc, MNS/Gib, SBN/Ygl, SR/Jr, WKY/N, WKY/Gla, WKY/NCrl, WKY/NHsd and WAG/GSto-Icgn), 22 rat strains/substrains serve as control or experimental animals in the studies on various pathological conditions that have no relation to hypertension or aging (ACI/N, ACI/EurMcwi, BBDP/Wor, 
$\mathrm{BN}-\mathrm{Lx} / \mathrm{Cub}, \quad \mathrm{BN}-\mathrm{Lx} / \mathrm{CubPrin}, \mathrm{BN} / \mathrm{SsN}, \quad \mathrm{BUF} / \mathrm{N}$, DA/BklArbNsi, F334/N, F344/NHsd, F344/NCrl, SUO_F344, GK/Ox, LE/Stm (SOLiD), LEW/Crl, LEW/NCrlBR, LE/Stm (Illumina), M520/N, MR/N, WAG/Rij, WN/N and Wistar) and 12 strains and substrains manifestated signs of hypertension (FHH/EurMcwi, LH/MavRrrc, MHS/Gib, SBH/Ygl, SHR/OlaIpcv, SHRSP/Gla, SHR/NCrlPrin, SHR/NHsd, SHR/OlaIpcvPrin, SS/Jr, SS/JrHsdMcwi and ISIAH/Icgn).

Functional enrichment analyses for the list of genes with SNPs was performed with DAVID tool (https://www.davidd.ncifcrf.gov/). List of the quantitative trait loci (QTLs) overlapped with the SNPs position was received using the RGD (https://rgd.mcw.edu/).

The identity by state (IBS) analysis of the alleles performed by SNPRelate in the R Software Environment was used to measure the distances between objects for dendrogram construction and for demonstrating the results of principal coordinates.

\section{Results}

A comparison of transcriptome sequences of the prefrontal cortex, hippocampus, and retina of OXYS rats with the reference genome of BN/NHsdMcwi rats revealed 42,478 SNPs in 9,903 genes. Analysis of the genetic similarity based on the identified SNPs showed that OXYS/Icgn rats are genetically closer to the ISIAH/Icgn rat strain, which was also developed from Wistar/Icgn rats, and two strains of Wistar Albino Glaxo rats (WAG/GSto-Icgn and WAG/Rij) than to 41 strains and substrains taken in the analysis.

Among the 42,478 SNPs found in OXYS rats, 40,373 SNPs in 9,699 genes have also been detected in the genomes of normotensive rat strains or strains used in studies on various pathological conditions that have no relation to hypertension or age-related diseases. Group of the remaining 2,105 SNPs includes 725 polymorphisms, which are found in both OXYS rats and in one or more hypertensive rat strains/substrains taken in analysis, as well as 1,380 polymorphisms that are specific to OXYS rats.

Using multidimensional scaling, a similarity between the genotypes of OXYS rats and 12 strains/substrains of hypertensive rats was evaluated. The data obtained show the similarity of the genotypes of OXYS and ISIAH/Icgn rats and their significant differences from the genotypes of strains and substrains of other hypertensive animals. Indeed, among the 725 SNPs that were detected in the hypertensive strains/substrains and OXYS rats, 663 SNPs were common to OXYS/Icgn rats and ISIAH/Icgn rats and were not found in other hypertensive rats. No common SNP was found for all hypertensive rat strains and substrains taken in the analysis. The maximum frequency of occurrence of SNPs detected in hypertensive strains/substrains was eight out of thirteen, with 4 out of this 8 strains represented by the substrains of SHR rats. This result indicates the differences in the genetic basis of different forms of hypertension.

A functional annotation of genes with SNPs common to OXYS rats and one or more hypertensive rat strains revealed enrichment in such functional groups as GTP/ATP binding, various signaling systems and cell division. Genes with SNPs specific for OXYS rats are associated with synapses/intercellular contacts, kinases, ATP binding, various signaling systems, $\mathrm{MHC}$, infection response and calcium binding. Many of those categories in both group involved in the development of hypertension according to various studies.
17 and 23 SNPs from those of 725 and 1380, respectively, presumably have significant effect on mRNA structure or protein function, that we described in detail in our previous work [2]. Among them the Ephxl gene annotated in RGD as associated with hypertension. In addition, Ephxl, as well as several other genes (Pla2r1, Zmym6, Trappc9, Nqo2) are associated with neurodegenerative diseases and/or mental disorders. Harmful polymorphisms are located in 313 and 451 QTLs. The most common parameters associated with these QTLs are blood pressure, bone structure and strength, bone mineral density, body weight, cardiac mass, kidney mass, diabetes mellitus, renal function, serum cholesterol and corticosterone levels, anxiety response.

\section{Conclusion}

Transcriptome of OXYS rats contains 725 common SNPs with one or more hypertensive rat strains and substrains. Analysis of genes with SNPs showed that only one gene Ephxl annotated in RGD as associated with hypertension. In addition, Ephx1, as well as several other genes (Pla2r1,Zmym6, Trappc9, Nqo2) are associated with neurodegenerative diseases and/or mental disorders. Multidimensional scaling analysis showed that OXYS rats are genetically distant from other strains and presumably have their own basis for the development of hypertension, which may determine the absence of senile phenotype of OXYS rats in the hypertensive rat strains.

\section{ACKNOWLEDGMENT}

This work was supported by the Budget Project (\# 02592019-0002)

\section{REFERENCES}

[1] Kolosova, N.G., Stefanova, N.A., Korbolina, E.E., Fursova, A.Zh., Kozhevnikova, O.S. The Senescence-Accelerated Oxys Rats--A Genetic Model of Premature Aging and Age-Dependent Degenerative Diseases. Adv Gerontol. 2014, 27(2), 336-340.

[2] Devyatkin, V.A., Redina, O.E., Kolosova, N.G., Muraleva, N.A. SingleNucleotide Polymorphisms Associated with the Senescence-Accelerated Phenotype of OXYS Rats: A Focus on Alzheimer's Disease-Like and Age-Related-Macular-Degeneration-Like Pathologies. J Alzheimers Dis. 2020, 73(3), 1167-1183. https://doi.org/10.3233/JAD-190956. 